\title{
Osteopathie bei gastroösophagealem Reflux mit Hiatushernie: Ein Fallbericht gemäß der CARE-Leitlinie
}

\author{
Gabriele Rotter Benno Brinkhaus \\ Institut für Sozialmedizin, Epidemiologie und Gesundheitsökonomie, Charité - Universitätsmedizin Berlin, Berlin, Deutschland
}

\section{Schlüsselwörter}

Hiatushernie - Gastroösophagealer Reflux .

Komplementärmedizin · Osteopathie

\section{Zusammenfassung}

Hintergrund: Das Vorhandensein einer Hiatushernie kann das Auftreten einer gastroösophagealen Refluxerkrankung (GERD) als Komplikation bedingen. Konventionelle medizinische Therapiemaßnahmen können zu unerwünschten Ereignissen und Rezidiven führen. Bisher sind die Effekte von osteopathischen Behandlungen bei Hiatushernie und GERD nicht bekannt. Fallbericht: Eine 59-jährige Patientin mit endoskopisch diagnostizierter chronischer Gastritis, GERD und Hiatushernie beklagte einen persistierenden gastroösophagealen Reflux trotz konventionell-medizinischer konservativer Therapie. Die osteopathische Diagnostik ergab eine funktionelle Störung im Bereich des Magens und der Kardia mit einer Beteiligung zugehöriger Reflexzonen. Nach einer osteopathischen Behandlung als individuelle, befundorientierte Therapie ließen die Beschwerden erheblich nach. Die Hiatushernie war nach einer dieser Behandlung endoskopisch nicht mehr nachweisbar. Schlussfolgerungen: Dieser Fallbericht schildert die Symptomreduktion einer GERD nach osteopathischer Behandlung. In der endoskopischen Folgeuntersuchung fand sich die initial diagnostizierte Hiatushernie nicht mehr, diese Befund änderung könnte jedoch auf die unterschiedlichen Untersucher zurückgeführt werden. Prospektive kontrollierte klinische Studien sind notwendig, um den Stellenwert von osteopathischen Behandlungen bei GERD mit Hiatushernie zu untersuchen.

(c) 2017 The Author(s). Published by S. Karger GmbH, Freiburg

\section{Keywords}

Hiatal hernia - Gastroesophageal reflux disease . Complementary medicine - Osteopathic medicine

\section{Summary}

Osteopathic Treatment of Gastroesophageal Reflux Disease and Hiatal Hernia: A Case Report Following the CARE Guideline

Background: A hiatal hernia can lead to gastroesophageal reflux disease (GERD) as one of its complications. Conventional medical therapies might cause adverse events and relapse of symptoms. So far, the effects of osteopathic treatment in hiatal hernia and in GERD are unclear. Case Report: A 59-year-old female patient with endoscopically diagnosed chronic gastritis, GERD, and hiatal hernia suffered from persisting gastroesophageal reflux despite conventional medical conservative therapy. Osteopathic diagnosis showed functional disorders in the stomach and cardia region, including corresponding reflex zones. After one osteopathic treatment being an individual medical finding-oriented therapy, the complaints diminished. The hiatal hernia was no longer endoscopically detectable after this treatment. Conclusions: This case report describes a symptom relief in GERD after osteopathic treatment. The initially endoscopically diagnosed hiatal hernia could no longer be detected in the following endoscopic inspection, maybe due to interobserver-discrepancies. Prospective controlled clinical trials are necessary to verify the role of osteopathic treatment in GERD and hiatal hernia.

\section{KARGER \\ Fax +497614520714} Accessible online at:
www.karger.com/cmr
Then arger on Commercial-NoDerivatives 4.0 International License (CC BY-NCD 4.0) (hit/lu mified matein requires witten pernission.
Dr. med. Gabriele Rotter

Institut für Sozialmedizin, Epidemiologie und Gesundheitsökonomie,

Hochschulambulanz für Naturheilkunde Standort Mitte

Charité - Universitätsmedizin Berlin

Luisenstraße 57, 10117 Berlin, Deutschland

gabriele.rotter@charite.de 


\section{Einleitung}

Eine Hiatushernie kann durch eine gastroösophageale Refluxerkrankung (GERD) kompliziert werden. Die Prävalenz der GERD nimmt zu und beträgt aktuell in Europa etwa 28\% [1].

Eine symptomatische Hiatushernie kann operativ oder nichtoperativ behandelt werden. Medikamentöse Therapiemaßnahmen zielen auf die Minderung der Konsequenzen des gastroösophagealen Refluxes [2]. Protonenpumpeninhibitoren reduzieren die Symptomatik häufig, können aber unerwünschte Effekte, wie Kopfschmerzen $(<5 \%)$, Diarrhoe $(<5 \%)$, eine erhöhte Anfälligkeit für pathogene Darmkeime und eine Osteopenie, sowie seltene schwere Nebenwirkungen, wie eine akute interstitielle Nephritis und eine schwere reversible Hypomagnesiämie, bedingen [2, 3]. Chirurgische Verfahren sind bei symptomatischer Gleithernie, trotz Medikation persistierendem Reflux oder Husten sowie bei einer Unverträglichkeit von Protonenpumpeninhibitoren indiziert [2]. Dabei erfolgen die Rückverlagerung von Magenanteilen in das Abdomen sowie eine Wiederherstellung der anatomischen Voraussetzungen für die Funktion des gastroösophagealen Übergangs [2]. Der aktuelle operative Standard ist die laparoskopische Fundoplicatio [2]. Die hauptsächlichen Komplikationen einer Fundoplicatio sind Dysphagie und Völlegefühl, das Risiko für schwere Komplikationen oder Tod wird hingegen mit 1-2\% benannt [2]. Die Rezidivrate nach einer operativen Therapie ist hoch, so nehmen bis zu 30\% der Patienten innerhalb der nächsten 5 Jahre erneut Antazida ein. Rezidivoperationen und folgende Ösophagogastroskopien werden häufig durchgeführt [4]. Aktuelle Ergebnisse weisen auf eine hohe Remissionsrate sowohl unter Gabe eines Protonenpumpeninhibitors als auch unter laparoskopischer Therapie hin $[5,6]$. An Lebensstilveränderungen werden diätische Maßnahmen sowie bei nächtlichem Reflux das Hochstellen des Kopfteils des Betts um 15-20 cm empfohlen [7]. Die Evidenz für die Wirksamkeit von Maßnahmen zu Lebensstilveränderungen bei GERD ist generell gering [2].

Die Osteopathie versteht sich als eigenständiger medizinischer Ansatz. Sie geht auf den amerikanischen Arzt A.T. Still zurück [8]. Manuelle Behandlungstechniken zur Unterstützung von körpereigenen Heilungskräften zielen auf eine Verbesserung von physiologischen Funktionen; der Körper wird dabei als strukturelle und funktionelle Einheit betrachtet [8]. Störungen des oberen Gastrointestinaltrakts können mittels viszerosomatischer Reflexphänomene zu somatischen Schmerzen, palpablen paraspinalen Gewebeänderungen und vertebralen Restriktionen führen [9].

In der Literatur gibt es nach Kenntnis der Autoren bisher keine Hinweise auf eine erfolgreiche osteopathische Behandlung einer Hiatushernie. Hinsichtlich der Behandlung eines möglichen Symptoms einer Hiatushernie, der GERD, wurde in einer Fallstudie über eine Symptomlinderung nach 3-maliger osteopathischer Behandlung berichtet [10]. In einer ohne Peer-Review veröffentlichten Beobachtungsstudie wurden 25 Patienten mit endoskopisch gesicherter Refluxerkrankung jeweils 8-mal osteopathisch-befundorientiert behandelt. Unter anderem verbesserten sich gastrointestinale Symptome und die Lebensqualität [11].
Der folgende Fallbericht erfolgt in Anlehnung an die CARELeitlinie [12]. Die Patientin stimmte der Veröffentlichung ihres Falls nach der Lektüre des Artikelentwurfs zu.

\section{Fallbericht}

\section{Anamnese}

Eine 59-jährige Patientin kaukasischer Ethnizität mit vorbekannter chronischer Gastritis, GERD und endoskopisch diagnostizierter Hiatushernie stellte sich mit Dringlichkeit im Juli 2015 in der Hochschulambulanz für Naturheilkunde der Charité - Universitätsmedizin Berlin am Standort Mitte aufgrund von starken Beschwerden durch einen gastroösophagealen Reflux vor. Sie berichtete von einem seit 10 Tagen bestehenden Reflux von Speisen bis in den Mund. Bedingt durch die vorangegangene konventionell-medizinische Versorgung führte die Patientin bereits nächtliche Oberkörperhochlagerungen durch und nahm einen Protonenpumpeninhibitor ein. Eine weitere Gastroskopie war geplant. Die Patientin beklagte Schmerzen und Blockierungsgefühle im Bereich der Brustwirbelsäule. Der Nachtschlaf der gewohnheitsmäßigen Bauchschläferin war bei hochgestelltem Oberkörperteil des Betts durch Schmerzen in Höhe des thorakolumbalen Übergangs und der Brustwirbelkörper 6-9 erheblich beeinträchtigt.

Hinsichtlich der gastralen Vorgeschichte waren 2009 nach einer Gastroskopie mit anschließender histologischer Untersuchung eine distale Ösophagitis bei klaffender Hiatushernie (Abb. 1) sowie eine geringe, chronische, Helicobacter-pylori-negative Antrumgastritis mit Ektasie der Kapillaren diagnostiziert und ein Protonenpumpeninhibitor verordnet worden. Im Rahmen einer Gastroskopie war im Jahr 2012 aufgrund rezidivierender Refluxsymptomatik eine histologisch gesicherte chronisch gering aktive Refluxkarditis diagnostiziert worden. Weiterhin fand sich eine große axiale Gleithernie, aber kein Nachweis von Barrett-Schleimhaut (Abb. 2). Von der Zahnreihe gemessen befand sich der Zwerchfellhiatus auf $38 \mathrm{~cm}$, im Untersuchungsverlauf auf $39 \mathrm{~cm}$ Höhe. Die etwas ausgefranste Z-Linie fand sich auf $35 \mathrm{~cm}$, im Untersuchungsverlauf auf etwa $37 \mathrm{~cm}$ Höhe.

Die Eigenanamnese beinhaltete chronisch-rezidivierende Beschwerden an der zervikalen und lumbalen Wirbelsäule, eine psychotherapeutische Behandlung aufgrund einer Depression mit Erschöpfungssymptomatik im Jahr 2013 und ein Herpes zoster im Rumpfbereich im Jahr 2014. Bis auf den Protonenpumpeninhibitor nahm die Patientin keine Arzneimittel ein. Der Sozialanamnese zufolge war die Diplompädagogin ausreichend sozial integriert. Sie beging keinen Substanzmissbrauch. Die vegetative Anamnese war bis auf häufige Durchschlafstörungen unauffällig.

\section{Körperlicher Untersuchungsbefund}

Die Patientin wies einen guten Allgemeinzustand und einen Body-MassIndex von $19,3 \mathrm{~kg} / \mathrm{m}^{2}$ auf. Bei der körperlichen Untersuchung stellten sich eine mäßig verstärkte Brustwirbelsäulenkyphose, eine Schulterprotraktion sowie eine diskrete epigastrale Druckempfindlichkeit heraus. Weitere pathologische Befunde wurden nicht dokumentiert.

\section{Osteopathische Diagnostik}

Die osteopathische Diagnostik ergab eine funktionelle Störung im Bereich des Magens und der Kardia mit sympathisch-segmentaler Beteiligung der thorakalen Segmente 6-9, eine Restriktion des Os occipitale zum 1. Halswirbelkörper, eine Restriktion des 12. Brustwirbelkörpers zum 1. Lendenwirbelkörper sowie Hinweise auf eine funktionelle Restriktion des Mediastinum links im dorsalen Anteil.

\section{Therapie}

Die befundorientierte osteopathische Behandlung zielte auf die Reduktion der Refluxsymptomatik mittels der Beeinflussung von dieser Symptomatik zugeordneten funktionellen Störungen. Angewendet wurden Techniken zur faszialen Dehnung des Ösophagus, zur Mobilisierung und Kaudalisierung der Kardia, zur reflektorischen Funktionsverbesserung betroffener Wirbelsäulen- 


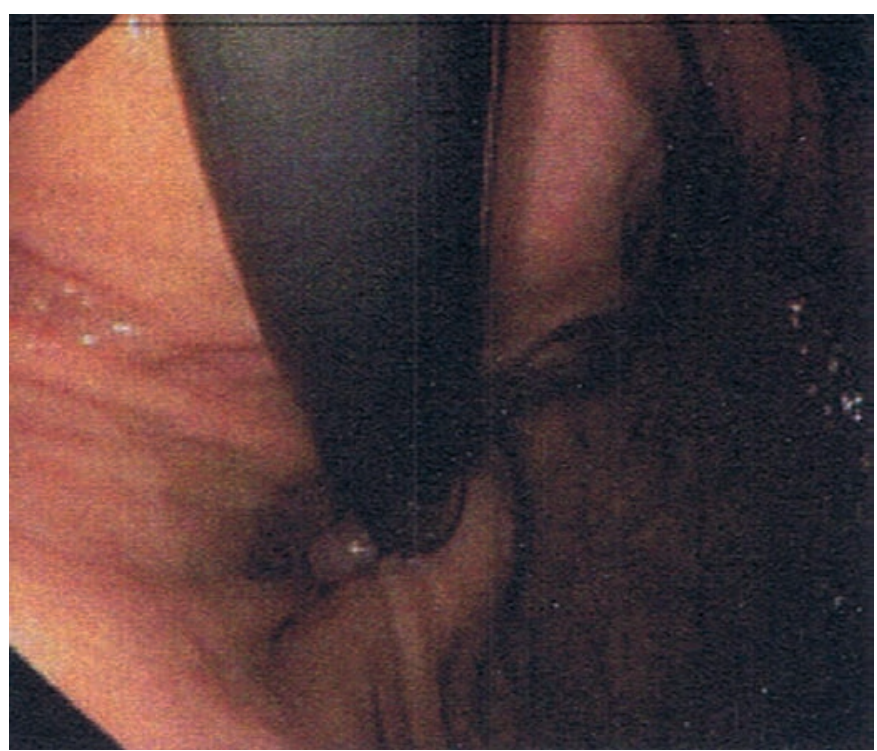

Abb. 1. Die klaffende Hiatushernie, 2009.

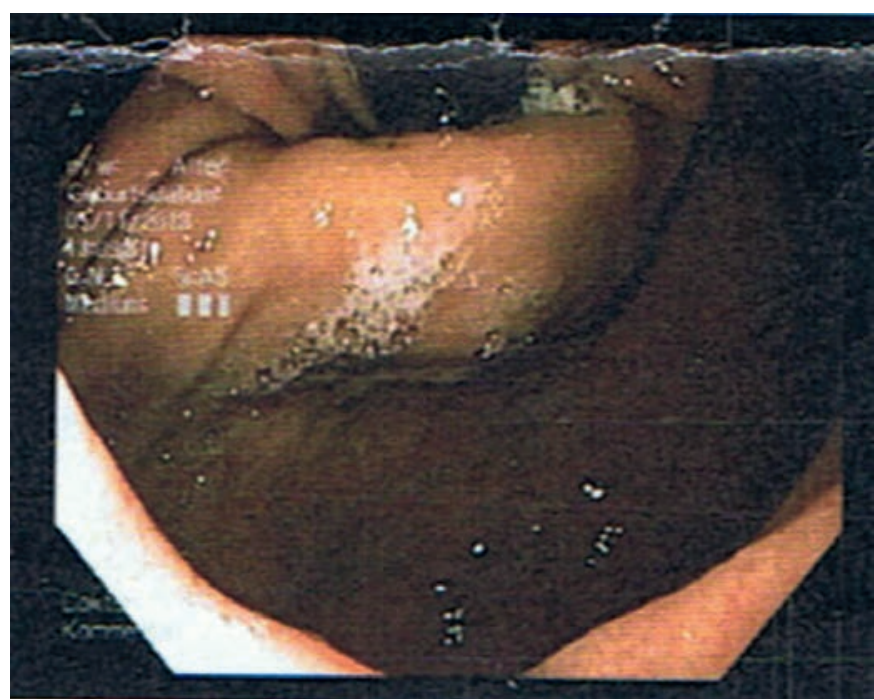

Abb. 2. Die klaffende Hiatushernie, 2012.

abschnitte, Muskelenergietechniken zur Verbesserung der segmentalen neuromuskulären Funktion sowie eine kraniosakrale Technik zum Lösen von faszialen diaphragmalen Spannungen. Die Patientin berichtete direkt nach der osteopathischen Behandlung von einer deutlichen Reduktion des thorakalen Blockierungsgefühls und von einer sofortigen Linderung der retrosternalen Missempfindung «Brennen» während der Ausführung der Technik «Dehnen des Ösophagus».

Nach 2 Wochen zeigte die Patientin insgesamt ein deutlich verbessertes Allgemeinbefinden mit anhaltendem Sistieren der Refluxsymptomatik ohne Einnahme von Antazida. In der zwischenzeitlich durchgeführten Gastroskopie zeigte sich keine Hiatushernie, bei regelrechtem Kardiaschluss (Abb. 3), jedoch eine histologisch gesicherte diskrete Refluxösophagitis ohne Erosionen (Abb. 4). In der histologischen Untersuchung fanden sich vereinzelte alzianophile Becherzellen. Diese verwiesen auf eine beginnende Barrett-Metaplasie ohne endoskopischen oder histologischen Befund einer Barrett-Schleimhaut.

Die osteopathische Untersuchung ergab rückläufige funktionelle Störungen im Bereich des Magens und der Kardia mit sympathisch-segmentaler Beteiligung von thorakalen Segmenten. Befundorientiert wurden Techniken zur faszialen Dehnung des Ösophagus, zur Persistenz der Kaudalisierung der

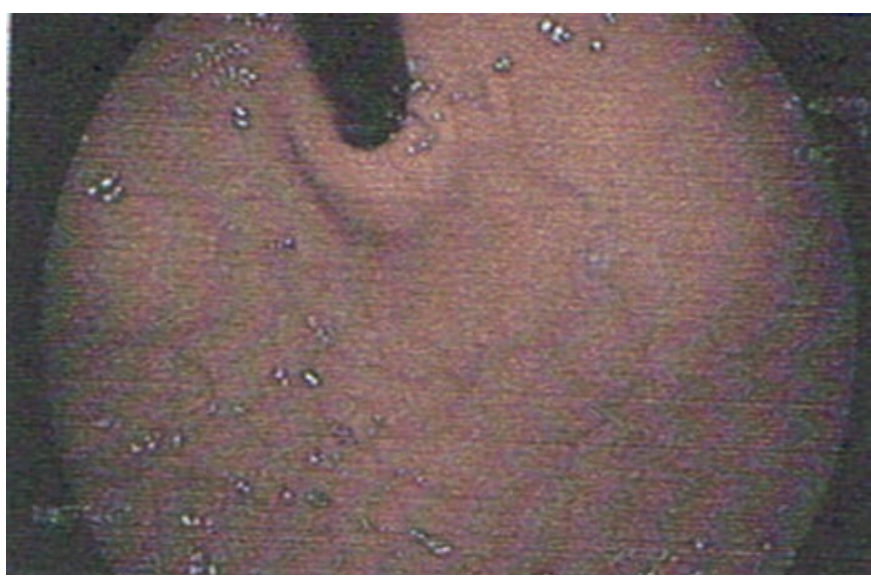

Abb. 3. Die Kardia schließt regelrecht, keine Hiatushernie, 2015.

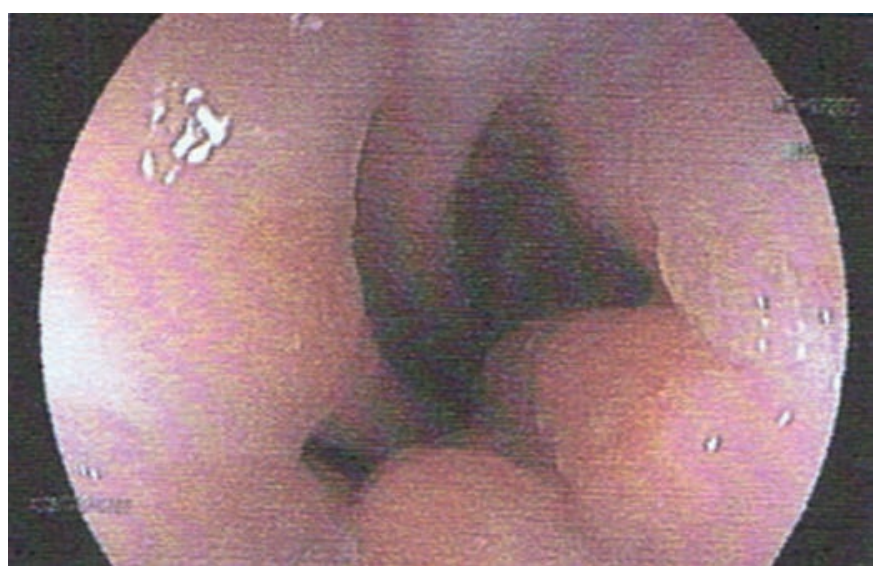

Abb. 4. Gastroösophagealer Übergang, keine Erosionen.

Kardia, zur reflektorischen Funktionsverbesserung betroffener thorakaler Wirbelsäulenabschnitte sowie kraniosakrale Techniken zur neurovegetativen Harmonisierung angewendet. Nach 8 Monaten berichtete die Patientin über eine anhaltende Symptomlinderung. Sie verneinte auf explizite Nachfrage unerwünschte Reaktionen auf die osteopathische Behandlung.

\section{Diskussion}

Der vorliegende Fallbericht schildert eine Symptomreduktion bei GERD bereits nach 1 osteopathischen Behandlung und das endoskopisch beschriebene Sistieren einer Hiatushernie. Bei einer Literaturanalyse fanden sich keine Fälle des Sistierens einer Hiatushernie unter nichtoperativer Therapie oder von deren Spontanremissionen im Erwachsenenalter.

Die Patientin litt trotz konventionell-medizinischer Therapie an persistierendem Reflux von Speisen bei einer Hiatushernie. Zusätzlich bestand bei der Patientin eine thorakale Kyphosierung. Die kausalen Zusammenhänge des häufigen gemeinsamen Auftretens von Kyphoskoliose und Hiatushernie sind noch nicht bekannt [13, 14].

Vertebrale und paravertebrale reflexbasierte Gewebeveränderungen der Brustwirbelsäule können funktionell bedingt sein, jedoch ebenfalls im Zusammenhang mit strukturellen Veränderungen von Ösophagus und Kardia stehen [15]. Die osteopathische 
Diagnostik der Patientin ergab eine funktionelle Störung im Bereich des Magen und der Kardia mit sympathisch-segmentaler Beteiligung vertebraler Segmente. Bereits 1 befundorientierte osteopathische Behandlung führte zu einer deutlichen Symptomlinderung, die durch eine zweite Behandlung konsolidiert wurde. Die Behandlung fokussierte auf die Kardia und ihre diaphragmale lokal-anatomische Umgebung; dabei wurde eine Kaudalisierung der Kardia angestrebt. Die Gründe für die sofortige Beschwerdelinderung während der osteopathischen Behandlung könnten sowohl in der tatsächlichen Dehnwirkung auf den Ösophagus als auch in neurovegetativen Phänomenen oder in einer tatsächlichen anatomischen Repositionierung liegen.

Ein begutachtender außenstehender Endoskopiker sichtete alle 3 Befundberichte der Gastroskopien und diagnostizierte im Befund von 2015 keine Kardiainsuffizienz (in Inversion) oder Hernie. Jedoch wies er auf die eingeschränkte Vergleichbarkeit der Befunde durch fehlende Angaben der Distanzen zur Z-Linie bei 2 der 3 Gastroskopien hin.

Aus konventionell-medizinischer Sicht besteht für die Wirksamkeit oder Möglichkeit einer Reposition der Kardia durch manuelle Techniken kein Nachweis. Der gastroösophageale Übergang stellt eine komplexe valvuläre Struktur dar, die den Reflux von Mageninhalt verhindert. Er besteht aus dem unteren Ösophagussphinkter, der physiologisch im Zwerchfellhiatus lokalisiert ist. Die Pars lumbalis diaphragmatis mit ihren Crura diaphragmatica übt in diesem Bereich eine zusätzliche sphinkterähnliche Kompression aus [16]. Die Barrierefunktion des gastroösophagealen Übergangs wird durch den intrinsischen Druck und die extrinsische Kompression des Ösophagussphinkters, die intraabdominale Lage des unteren Ösophagussphinkters, die Intaktheit des phrenoösophagealen Ligaments und den Erhalt des spitzen Winkels zwischen Ösophagus und Magen bedingt [17]. Die pathologischen Voraussetzungen zahlreicher Symptome der GERD sind bisher nicht vollständig geklärt [18]. Bekannt ist, dass der Reflux von Magensäure häufig als Sodbrennen wahrgenommen wird. Der gastroösophageale Reflux kann jedoch ebenfalls durch einen Gallereflux, eine Erweiterung des Magens oder des Ösophagus und durch Motilitätsstörungen hervorgerufen werden [18]. Weiterhin gibt es Hinweise auf mögliche funktionelle Ursachen von Missempfindungen bei gastroösophagealem Reflux. Anhaltende longitudinale Kontraktio- nen der Ösophagusmuskulatur können mit nachfolgenden thorakalen Schmerzen und Sodbrennen in Verbindung gebracht werden [19]. Es wird vermutet, dass diese Kontraktionen über eine Ischämie der Ösophaguswand zu diesen Beschwerden führen können [20]. Weitere Mechanismen der Entwicklung und Perzeption der Symptomatik eines gastroösophagealen Refluxes, einschließlich peripherer und zentraler Sensibilisierungsmechanismen, werden diskutiert [21].

\section{Stärken und Limitationen}

Dieser Fallbericht schildert erstmals eine Symptomreduktion bei GERD nach 1 osteopathischen Behandlung bei gleichzeitigem endoskopisch beschriebenem Sistieren einer Hiatushernie. In den bisher beschriebenen Fällen erfolgte eine Symptomlinderung der GERD nach 3- bis 8-maliger osteopathischer Behandlung [10, 11]. Einschränkend muss konstatiert werden, dass die 3 Gastroskopien bei dieser Patientin von unterschiedlichen Untersuchern durchgeführt wurden. Die Limitationen dieses Fallberichts bestehen in möglichen «Interobserver»-Differenzen hinsichtlich des Vorliegens/Sistierens der Hiatushernie.

\section{Schlussfolgerung}

Der vorliegende Fallbericht schildert die Symptomreduktion einer GERD nach osteopathischer Behandlung. In der endoskopischen Folgeuntersuchung fand sich die initial diagnostizierte Hiatushernie nicht mehr. Diese Befundänderung könnte jedoch auf die unterschiedlichen Untersucher zurückgeführt werden. Prospektive kontrollierte klinische Studien einschließlich endoskopischer Befundkontrollen über einen Langzeitverlauf sind notwendig, um den Stellenwert von osteopathischen Behandlungen bei GERD mit Hiatushernie zu untersuchen.

\section{Disclosure Statement}

Die Autoren erklären, dass keine Interessenkonflikte in Bezug auf den vorliegenden Artikel bestehen.

\section{Literatur}

1 El-Serag HB, Sweet S, Winchester CC, Dent J: Update on the epidemiology of gastro-oesophageal reflux disease: a systematic review. Gut 2014;63:871-880.

2 Roman S, Kahrilas PJ: The diagnosis and management of hiatus hernia. BMJ 2014;349:g6154.

3 Yang YX, Metz DC: Safety of proton pump inhibitor exposure. Gastroenterology 2010;139:1115-1127.

4 Vakil N, Shaw M, Kirby R: Clinical effectiveness of laparoscopic fundoplication in a U.S. community. Am J Med 2003;114:1-5.
5 Hatlebakk JG, Zerbib F, Bruley des Varannes S, Attwood SE, Ell C, Fiocca R, Galmiche JP, Eklund S, Langstrom G, Lind T, Lundell LR; on Behalf of the LOTUS Study Group: Gastroesophageal acid reflux control 5 years after antireflux surgery, compared with long-term esomeprazole therapy. Clin Gastroenterol Hepatol 2016;14:678-685.

6 Galmiche JP, Hatlebakk J, Attwood S, Ell C, Fiocca R, Eklund S, Langstrom G, Lind T, Lundell L: Laparoscopic antireflux surgery vs esomeprazole treatment for chronic GERD: the LOTUS randomized clinical trial. JAMA 2011;305:1969-1977.
7 Kahrilas PJ, Shaheen NJ, Vaezi MF, Hiltz SW, Black E, Modlin IM, Johnson SP, Allen J, Brill JV; American Gastroenterological Association: American Gastroenterological Association Medical Position Statement on the management of gastroesophageal reflux disease. Gastroenterology 2008;135:1383-1391, 1391.e1381-1385.

8 World Health Organization: Benchmarks for Training in Osteopathy: Benchmarks for training in traditional/ complementary and alternative medicine. Geneva, World Health Organization, 2010.

9 Kuchera M: Osteopathic Considerations in Systemic Dysfunction. [S.l.], Greyden Press, 1996. 
10 Diniz LR, Nesi J, Curi AC, Martins W: Qualitative evaluation of osteopathic manipulative therapy in a patient with gastroesophageal reflux disease: a brief report. J Ame Osteopath Assoc 2014;114:180-188.

11 Nerreter A, Ruf S, Höly B: Osteopathische Behandlung bei gastroösophagealem Reflux. Eine Beobachtungsstudie. COE, 2006.

12 Gagnier JJ, Kienle G, Altman DG, Moher D, Sox H, Riley D; CARE Group: The CARE guidelines: consensus-based clinical case reporting guideline development. Glob Adv Health Med 2013;2:38-43.

13 Polomsky M, Siddall KA, Salvador R, Dubecz A, Donahue LA, Raymond D, Jones C, Watson TJ, Peters JH: Association of kyphosis and spinal skeletal abnormalities with intrathoracic stomach: a link toward understanding its pathogenesis. J Am Coll Surg 2009;208: 562-569.
14 Polomsky M, Peters JH, Schwartz SI: Hiatal hernia and disorders of the spine: a historical perspective. Dis Esophagus 2012;25:367-372.

15 Snider KT, Schneider RP, Snider EJ, Danto JB, Lehnardt CW, Ngo CS, Johnson JC, Sheneman TA: Correlation of somatic dysfunction with gastrointestinal endoscopic findings: an observational study. J Am Osteopath Assoc 2016;116:358-369.

16 Kwiatek MA, Kahrilas PJ: Physiology of the LES. Dis Esophagus 2012;25:286-291.

17 Pandolfino JE, Kwiatek MA, Kahrilas PJ: The pathophysiologic basis for epidemiologic trends in gastroesophageal reflux disease. Gastroenterol Clin North Am 2008;37:827-843, viii.

18 Mikami DJ, Murayama KM: Physiology and pathogenesis of gastroesophageal reflux disease. Surg Clin North Am 2015;95:515-525.
19 Balaban DH, Yamamoto Y, Liu J, Pehlivanov N, Wisniewski R, DeSilvey D, Mittal RK: Sustained esophageal contraction: a marker of esophageal chest pain identified by intraluminal ultrasonography. Gastroenterology 1999;116:29-37.

20 Pehlivanov N, Liu J, Mittal RK: Sustained esophageal contraction: a motor correlate of heartburn symptom. American journal of physiology Am J Physiol Gastrointest Liver Physio 2001;281:G743-751.

21 Herregods TVK, Bredenoord AJ, Smout AJPM: Pathophysiology of gastroesophageal reflux disease: new understanding in a new era. Neurogastroenterol Motil 2015;27:1202-1213 